DR-26

\title{
PYRENE-DERIVED GRIGNARD REAGENT(S): PREPARATION AND USE IN KEY CARBONYLATION/CARBOXYLATION REACTIONS
}

\author{
O. S. Taniya, ${ }^{1,2}$ L. K. Sadieva, ${ }^{1}$ S. Santra, ${ }^{1}$ A. F. Khasanov, ${ }^{1,2}$ M. Rahman, ${ }^{1}$ I. S. Kovalev, ${ }^{1}$ D. S. \\ Kopchuk, ${ }^{1,2}$ G. V. Zyryanov, ${ }^{1,2}$ V. L. Rusinov, ${ }^{1,2}$ O. N. Chupakhin ${ }^{1,2}$
}

${ }^{1}$ Ya. Postovsky Institute of Organic Synthesis UB RAS, S. Kovalevskoy/Akademicheskaya St., 20/22, Yekaterinburg, 620990, Russia.

${ }^{2}$ Ural Federal University of the first President of Russia B. N. Yeltsin. 19 Mira St. Yekaterinburg,620002,Russia. E-mail: olgataniya@yandex.ru \begin{abstract}
to develop new methods for the surface functionalization[5]. obtain pyrenecontaining compounds with desired properties(Fig.1).

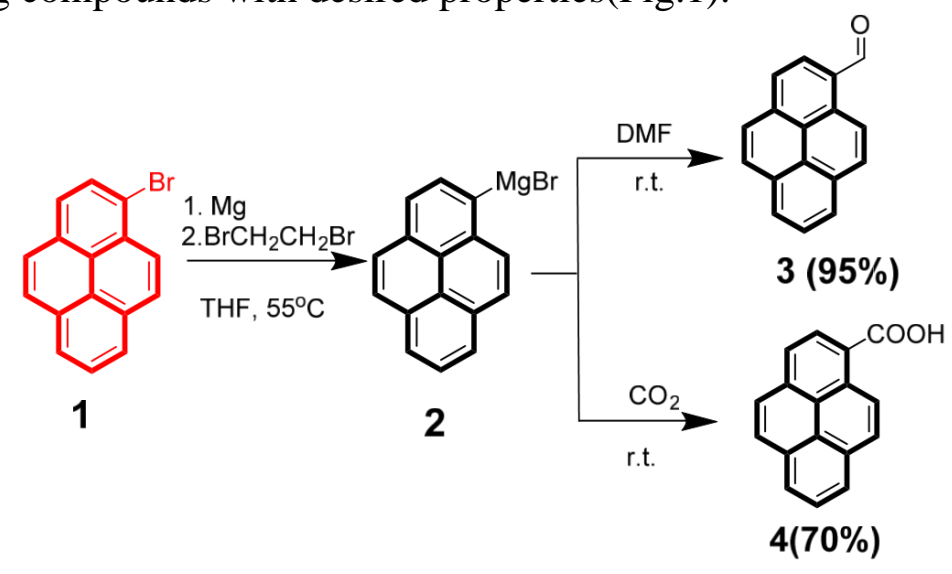

Abstract. Among traditional pyrene-containing derivatives, carbonyl/carboxyl compounds are important organic dyes/components, and their photophysical properties have been well studied[1]. Due to the high photoluminescence quantum yield[2] and the ability to form stable donor-acceptor complexes with electron deficient compounds, including nitroaromatic ones[3], pyrene derivatives are used as monomolecular chemosensors for the detection of nitro-explosives in solutions[4]. For the producing advanced materials, for instance based on graphene/graphene oxide, pyrenecarboxylic acid is widely used

We have developed the atom-economical synthesis of pyrene-1-carbaldehyde 3, pyrene1carboxylic acid 4 through 1-pyrenmagnesium bromide (Grignard reagent) derived from 1bromopyrene[6] with moderate to high yields under mild conditions, as important synthons in order to

Figure 1. Scheme of synthesis of pyrene-1-carbaldehyde 3 and pyrene-1-carboxylic acid 4

The chemical structures of compounds 3-4 are confirmed by ${ }^{1} \mathrm{H}$ and ${ }^{13} \mathrm{C}$ NMR spectroscopy, mass spectrometric data and elemental analysis.

\section{References}

1. Niko Y. et al. Fundamental photoluminescence properties of pyrene carbonyl compounds through absolute fluorescence quantum yield measurement and density functional theory // Tetrahedron. Elsevier Ltd, 2012. Vol. 68, № 31. P. 61776185 .

2. Berlman I.B. Handbook of fluorescence spectra of aromatic molecules. Academic Press, 1971. 473 p.

3. Barnes J.C. et al. Complexes of pyrene with 2,4,6-trinitroanisole. Studies of association in solution and the crystal structure of the 1:1 complex // Tetrahedron. 1984. Vol. 40, № 9. P. 1595-1601.

4. Kovalev I.S. et al. Fluorescent Detection of 2,4-DNT and 2,4,6-TNT in Aqueous Media by Using Simple Water-Soluble Pyrene Derivatives // Chem. - An Asian J. John Wiley \& Sons, Ltd, 2016. Vol. 11, № 5. P. 775-781.

5. Prasad C., et al. An overview of graphene oxide supported semiconductors based photocatalysts: Properties, synthesis and photocatalytic applications // J. Mol. Liq. Elsevier, 2019. P. 111826.

6. Mitchell R.H., Lai Y.-H., Williams R. V. N-Bromosuccinimide-dimethylformamide: a mild, selective nuclear monobromination reagent for reactive aromatic compounds // J. Org. Chem. 1979. Vol. 44, № 25. P. $4733-4735$. 